\title{
Severe acute cytomegalovirus hepatitis in an immunocompetent child: Case report
}

Authors: Gh. Jugulete ${ }^{1,2}$, Endis Osman ${ }^{1}$, Mădălina Merișescu ${ }^{1,2}$, Elena Gheorghe ${ }^{1}$, Andreea Madalina Panciu ${ }^{1}$

${ }^{I}$ National Institute of Infectious Disease "Prof. Dr. Matei Balș" Bucharest

${ }^{2}$ University of Medicine and Pharmacy "Carol Davila" Bucharest

\section{Introduction}

- The clinical course of the infection is usually mild, although a small percentage of patients suffer from protracted and fever.

- CMV infection in immunocompetent hosts may rarely be able to lead to severe organ specific complications.

- Most cases of CMV induced hepatitis occur in adults with severe immune deficiency.

- Only a few cases involving immunocompetent patients have been reported. Severe hepatitis is an uncommon presentation.

\section{Material and methods}

We present the case of a 6-year-old child presented with a 1-week history of recurrent fever and nonspecific maculo-papular rash.

- Her initial evaluation physical revealed fever, angina and moderate hepatosplenomegaly.

- Laboratory tests showed newly increased transaminase activity ( $x$ $300 N$ ) and serum bilirubin and prothrombin time were also severely impaired. She was admitted for evaluation of acute hepatitis in the 9th Paediatric Departamen at the National Institute for Infectious Diseases „Pof. Dr. Matei Bals” - Bucharest, Romania.

\section{Results}

- Serology for hepatitis A, B, C, E and HIV were negative.

- Abdominal imaging indicated moderate hepatosplenomegaly.

- Cultures were sterile. Additional tests for uncommon viral hepatitis included herpes simplex virus, cytomegalovirus and Epstein-Barr virus.

- Subsequently, cytomegalovirus serology showed an initial IgM positive and negative IgG titre and repeated titres of cytomegalovirus serology showed seroconversion.
- Cytomegalovirus DNA qualitative PCR was negative in the day 10 after the onset of the simptome.

- No antiviral medication was given, but she required fresh plasma suport for the coagulation impairement.

- She continued to have intermittent daily fever but reported no associated symptoms.

- She was discharged $\mathbf{1 0}$ days after admission in good condition, her serum hepatic profile returned to normal and she reported no more episodes of fever or rash.

\section{Conclusions}

- Infection with cytomegalovirus is an unusual cause of acute infectious hepatitis in an immunocompetent patient.

- Thus, cytomegalovirus hepatitis should be considered in the differential diagnosis in immunocompetent patients presenting with non-specific prodromal symptoms and unexplained hepatitis. 